\title{
Neutron density distributions for atomic parity nonconservation experiments
}

\author{
D. Vretenar ${ }^{1,2}$, G.A. Lalazissis ${ }^{1,3}$, and P. Ring ${ }^{1}$ \\ 1 Physik-Department der Technischen Universität München, D-85748 Garching, Germany \\ ${ }^{2}$ Physics Department, Faculty of Science, University of Zagreb, 10000 Zagreb, Croatia \\ 3 Physics Department, Aristotle University of Thessaloniki, Thessaloniki GR-54006, Greece
}

\begin{abstract}
The neutron distributions of $\mathrm{Cs}, \mathrm{Ba}, \mathrm{Yb}$ and $\mathrm{Pb}$ isotopes are described in the framework of relativistic mean-field theory. The self-consistent ground state proton and neutron density distributions are calculated with the relativistic Hartree-Bogoliubov model. The binding energies, the proton and neutron radii, and the quadrupole deformations are compared with available experimental data, as well as with recent theoretical studies of the nuclear structure corrections to the weak charge in atomic parity nonconservation experiments.
\end{abstract}

PACS number(s): 32.80.Ys, 12.15.Ji, 21.10.Gv, 21.60.-n 


\section{INTRODUCTION}

Measurements of parity nonconservation (PNC) effects in intermediate and heavy atomic systems might provide very stringent tests of the Standard model of electroweak interactions. Nuclei and electrons are bound into atoms by electromagnetic interactions that do not violate parity. Parity nonconservation arises from the interference of the $\gamma$ and $Z^{0}$ gauge boson exchange between the nucleus and the atomic electrons. The dominant part of the PNC interaction results from the coupling of the axial electronic current to the vector nuclear current, and it allows normally forbidden transitions. The nuclear vector current is conserved and the PNC effects provide a measure of the electroweak coupling of the elementary quarks. An extensive analysis of the sensitivity of a variety of low-energy PNC observables to possible new physics phenomena has been recently reported in Ref. [1].

The nucleus acts as a source of the weak charge $Q_{W}$. The quantity which is measured in experiments is the electric dipole amplitude between two electronic states which, in the absence of the PNC electron-nucleus interaction, would have the same parity. This observable can be parameterized [1, 2]

$$
\epsilon_{P N C}=\xi Q_{W}=\xi\left[Q_{W}^{S t . M o d}+\Delta Q_{W}^{n-p}\right]
$$

where $\xi$ is a coefficient that depends on atomic structure, $Q_{W}^{S t . M o d}$ is the Standard model prediction for the weak charge, and $\Delta Q_{W}^{n-p}$ results from the difference between the neutron and proton density distributions in the nucleus. At tree level

$$
Q_{W}^{S t . M o d}=\left(1-4 \sin ^{2} \theta_{W}\right) Z-N
$$

$\mathrm{Z}$ and $\mathrm{N}$ are the numbers of protons and neutrons, respectively, and $\theta_{W}$ is the weak mixing angle. The finite size of the nucleus modifies $Z \rightarrow q_{p} Z$ and $N \rightarrow q_{n} N$, where

$$
q_{n(p)}=\int f(r) \rho_{n(p)}(r) d^{3} r
$$

The function $f(r)$ describes the spatial variation of the electron axial transition matrix element over the nucleus. The difference between the neutron $\rho_{n}$ and proton $\rho_{p}$ density distributions modifies the effective weak charge 


$$
\Delta Q_{W}^{n-p}=N\left(1-q_{n} / q_{p}\right) .
$$

The two principal factors, therefore, which limit the precision with which the weak charge $Q_{W}$ can be determined from atomic experiments are: (i) the accuracy of atomic theory calculations ( $\xi$ in Eq. (1) and the function $f(r)$ in Eq. (3)), and (ii) the nuclear structure effects, i.e. the uncertainties in the neutron and proton density distributions. At present, the level of accuracy of atomic structure calculations constitutes the predominant uncertainty in the interpretation of atomic PNC observables [四]. For example, the most accurate experimental result for the weak charge of the Cs nucleus is $Q_{W}^{\text {expt }}=-72.06(28)_{\text {expt }}(34)_{\text {theory }}$ [3]. The Standard model value including radiative corrections is $Q_{W}^{S t . M o d}=-73.20(13)$. It has been suggested [1] that the measurement of PNC effects along an isotope chain might represent a useful method to circumvent the atomic theory uncertainty. The ratios of the electric dipole amplitudes for different isotopes should not depend on the details of atomic structure. In addition to the obvious $\mathrm{N}$ dependence, these ratios will be sensitive to the value of the Weinberg angle and, eventually, to additional parameters of a more general electroweak theory [1,5]. The shortcoming of this method, however, is that the ratios of PNC observables will also be sensitive to changes in the neutron distribution $\rho_{n}$ along the isotope chain. The PNC interaction is directly proportional to the overlap between electrons and neutrons. And while extremely accurate data on proton distributions in nuclei are obtained from electron scattering experiments, data of comparable precision on neutron density distributions are presently not available. It is much more difficult to measure the distribution of neutrons, though more recently accurate data on differences in radii of the neutron and proton density distributions have been obtained [6:7].

A number of studies have been reported on the nuclear structure effects in the interpretation of atomic $\mathrm{PNC}$ experiments in $\mathrm{Cs}$ and $\mathrm{Pb}$ isotope chains. The basic theoretical framework has been analyzed in Ref. [5], and it was pointed out that the uncertainties in the neutron distributions would have to be significantly reduced, if isotope ratios are to be used in high-precision evaluation of the atomic PNC parameters. On the other hand, it was 
also emphasized that atomic PNC experiments might provide a model independent tool for studying variations in neutron distributions. In a more extensive analysis of electroweak parameters and nuclear structure effects [8], a number of relativistic and nonrelativistic nuclear model calculations were performed for the chain of $\mathrm{Pb}$ isotopes. The conclusion was that the spread among model predictions for the sizes of nuclear structure effects may preclude using $\mathrm{Pb}$ isotope ratios to test the Standard model at better than a $1 \%$ level. The theoretical analysis included the Hartree-Fock-Bogoliubov model with Gogny forces, various Hartree-Fock Skyrme effective interactions, as well as the relativistic mean-field model. The Hartree-Fock Skyrme model, with the inclusion of deformation effects, was used in Ref. [9] to calculate the proton and neutron densities in the isotope chain ${ }^{125} \mathrm{Cs}-{ }^{139} \mathrm{Cs}$. The good agreement with the experimental charge radii, binding energies, and ground-state spins, led to the estimate that the uncertainties in the differences of the neutron radii may cause uncertainties in the ratio of weak charges of less than $10^{-3}$, safely smaller than the anticipated experimental error. In a detailed analysis of Ref. [10] it was shown that extremely high-precision measurements of PNC effects in atomic $\mathrm{Yb}$ appear possible, and it was suggested that a comparison of PNC observables in the wide range of stable isotopes of $\mathrm{Yb}$ may provide a unique test of the Standard model. The recent experimental result for the weak charge of Cs [3] has prompted the analysis of the effects of neutron spatial distribution on atomic PNC in Cs [2]. Several theoretical models have been used to calculate the modifications to the nuclear weak charge due to small differences between the spatial distributions of neutrons and protons in the Cs nucleus. It has been shown that the nuclear structure uncertainties are smaller than those arising from atomic theory calculations, but are comparable to the present uncertainties in the Standard model prediction. The sensitivity of atomic PNC to nuclear structure effects in single isotopes and isotope chains has also been extensively discussed in Refs. [1] and [11].

In the comparison of the theoretical models that have been used in the analysis of nuclear structure effects, it has been pointed out [2, 8] that relativistic mean field models typically generate significantly larger neutron radii, and thus would predict much larger corrections to the nuclear weak charge. Models of nuclear structure based on relativistic hadrodynam- 
ics [12] have attracted much interest in the last decade. In particular, the relativistic meanfield model has been very successfully applied in the description of a large variety of nuclear structure phenomena in spherical and deformed nuclei [13. The model has more recently been extended to include both mean-field and pairing correlations in a unified framework: the relativistic Hartree-Bogoliubov model [14,15]. A unified and self-consistent description of the long range mean-field correlations and the pairing field is especially important for the structure of open shell nuclei further away from the line of $\beta$-stability. In addition, in the last few years several new and more accurate parameter sets of meson masses and mesonnucleon coupling constants for the effective mean-field Lagrangian have been derived. These new effective forces have been used not only for a more accurate description of properties of $\beta$-stable nuclei, but they have also been applied in studies of isovector properties of exotic nuclei with large isospin values, of the structure of superheavy nuclei, and properties of excited states in the relativistic random-phase approximation and the time-dependent relativistic mean-field model.

In a recent study [16] we have applied the relativistic Hartree-Bogoliubov model to the description of parity-violating elastic electron scattering (PVES) on neutron-rich $\mathrm{Ne}, \mathrm{Na}, \mathrm{Ni}$ and $\mathrm{Sn}$ isotopes. For the elastic scattering of $850 \mathrm{MeV}$ electrons on these nuclei, the parityviolating asymmetry parameters have been calculated using a relativistic optical model with inclusion of Coulomb distortion effects. The asymmetry parameters for chains of isotopes have been compared, and their relation to the Fourier transforms of neutron densities has been studied. The important impact that PVES measurement of neutron densities could have on atomic PNC experiments has been recently discussed in Ref. [11]. Since the sensitivity of the neutron distribution appears to be approximately the same for PVES and atomic PNC, it would of course be ideal if density distributions for PNC experiments were determined from PVES. In the present work we use the relativistic Hartree-Bogoliubov model to calculate ground-state proton and neutron distributions for the isotopic chains that have been suggested for atomic PNC experiments: Cs, Ba, Yb and Pb. Calculated quantities will be compared with available experimental data, as well as with previous analyses of 
the nuclear structure corrections to the weak charge [2,8,8.9]. In Section II we present an outline of the relativistic Hartree-Bogoliubov model. The neutron densities for atomic PNC experiments are calculated and analyzed in Section III.

\section{THE RELATIVISTIC HARTREE-BOGOLIUBOV MODEL}

The relativistic mean field theory is based on simple concepts: nucleons are described as point particles, the theory is fully Lorentz invariant, the nucleons move independently in mean fields which originate from the nucleon-nucleon interaction. Conditions of causality and Lorentz invariance impose that the interaction is mediated by the exchange of point-like effective mesons, which couple to the nucleons at local vertices. The single-nucleon dynamics is described by the Dirac equation

$$
\left\{-i \boldsymbol{\alpha} \cdot \boldsymbol{\nabla}+\beta\left(m+g_{\sigma} \sigma\right)+g_{\omega} \omega^{0}+g_{\rho} \tau_{3} \rho_{3}^{0}+e \frac{\left(1-\tau_{3}\right)}{2} A^{0}\right\} \psi_{i}=\varepsilon_{i} \psi_{i} .
$$

$\sigma, \omega$, and $\rho$ are the meson fields, and $A$ denotes the electromagnetic potential. $g_{\sigma} g_{\omega}$, and $g_{\rho}$ are the corresponding coupling constants for the mesons to the nucleon. The lowest order of the quantum field theory is the mean-field approximation: the meson field operators are replaced by their expectation values. The sources of the meson fields are defined by the nucleon densities and currents. The ground state of a nucleus is described by the stationary self-consistent solution of the coupled system of the Dirac (5)and Klein-Gordon equations:

$$
\begin{aligned}
{\left[-\Delta+m_{\sigma}^{2}\right] \sigma(\mathbf{r}) } & =-g_{\sigma} \rho_{s}(\mathbf{r})-g_{2} \sigma^{2}(\mathbf{r})-g_{3} \sigma^{3}(\mathbf{r}) \\
{\left[-\Delta+m_{\omega}^{2}\right] \omega^{0}(\mathbf{r}) } & =g_{\omega} \rho_{v}(\mathbf{r}) \\
{\left[-\Delta+m_{\rho}^{2}\right] \rho^{0}(\mathbf{r}) } & =g_{\rho} \rho_{3}(\mathbf{r}) \\
-\Delta A^{0}(\mathbf{r}) & =e \rho_{p}(\mathbf{r}),
\end{aligned}
$$

for the sigma meson, omega meson, rho meson and photon field, respectively. Due to charge conservation, only the 3rd-component of the isovector rho meson contributes. The source terms in equations (6) to (9) are sums of bilinear products of baryon amplitudes, and they 
are calculated in the no-sea approximation, i.e. the Dirac sea of negative energy states does not contribute to the nucleon densities and currents. Due to time reversal invariance, there are no currents in the static solution for an even-even system, and therefore the spatial vector components $\boldsymbol{\omega}, \boldsymbol{\rho}_{\mathbf{3}}$ and $\mathrm{A}$ of the vector meson fields vanish. The quartic potential

$$
U(\sigma)=\frac{1}{2} m_{\sigma}^{2} \sigma^{2}+\frac{1}{3} g_{2} \sigma^{3}+\frac{1}{4} g_{3} \sigma^{4}
$$

introduces an effective density dependence. The non-linear self-interaction of the $\sigma$ field is essential for a quantitative description of properties of finite nuclei.

In addition to the self-consistent mean-field potential, pairing correlations have to be included in order to describe ground-state properties of open-shell nuclei. For nuclei close to the $\beta$-stability line, pairing has been included in the relativistic mean-field model in the form of a simple BCS approximation [17]. For more exotic nuclei further away from the stability line, however, the BCS model presents only a poor approximation. In particular, in order to correctly reproduce density distributions in neutron-rich nuclei, mean-field and pairing correlations have to be described in a unified framework: the Hartree-Fock-Bogoliubov model or the relativistic Hartree-Bogoliubov (RHB) model. In the unified framework the ground state of a nucleus $\mid \Phi>$ is represented by the product of independent single-quasiparticle states. These states are eigenvectors of the generalized single-nucleon Hamiltonian which contains two average potentials: the self-consistent mean-field $\hat{\Gamma}$ which encloses all the long range particle-hole $(p h)$ correlations, and a pairing field $\hat{\Delta}$ which sums up the particleparticle $(p p)$ correlations. In the Hartree approximation for the self-consistent mean field, the relativistic Hartree-Bogoliubov equations read

$$
\left(\begin{array}{cc}
\hat{h}_{D}-m-\lambda & \hat{\Delta} \\
-\hat{\Delta}^{*} & -\hat{h}_{D}+m+\lambda
\end{array}\right)\left(\begin{array}{l}
U_{k}(\mathbf{r}) \\
V_{k}(\mathbf{r})
\end{array}\right)=E_{k}\left(\begin{array}{c}
U_{k}(\mathbf{r}) \\
V_{k}(\mathbf{r})
\end{array}\right) .
$$

where $\hat{h}_{D}$ is the single-nucleon Dirac Hamiltonian (5), and $m$ is the nucleon mass. The chemical potential $\lambda$ has to be determined by the particle number subsidiary condition in order that the expectation value of the particle number operator in the ground state equals 
the number of nucleons. The column vectors denote the quasi-particle spinors and $E_{k}$ are the quasi-particle energies. The pairing field $\hat{\Delta}$ in (11) is defined

$$
\Delta_{a b}\left(\mathbf{r}, \mathbf{r}^{\prime}\right)=\frac{1}{2} \sum_{c, d} V_{a b c d}\left(\mathbf{r}, \mathbf{r}^{\prime}\right) \sum_{E_{k}>0} U_{c k}^{*}(\mathbf{r}) V_{d k}\left(\mathbf{r}^{\prime}\right)
$$

where $a, b, c, d$ denote quantum numbers that specify the Dirac indices of the spinors, $V_{a b c d}\left(\mathbf{r}, \mathbf{r}^{\prime}\right)$ are matrix elements of a general two-body pairing interaction. The RHB equations are solved self-consistently, with potentials determined in the mean-field approximation from solutions of Klein-Gordon equations for the meson fields. The current version of the model [18 describes axially symmetric deformed shapes. The Dirac-Hartree-Bogoliubov equations and the equations for the meson fields are solved by expanding the nucleon spinors $U_{k}(\mathbf{r})$ and $V_{k}(\mathbf{r})$, and the meson fields in terms of the eigenfunctions of a deformed axially symmetric oscillator potential [17]. The calculations for the present analysis have been performed by an expansion in 12 oscillator shells for the fermion fields, and 20 shells for the boson fields. A simple blocking procedure is used in the calculation of odd-proton and/or odd-neutron systems. The blocking calculations are performed without breaking the timereversal symmetry.

The input parameters of the RHB model are the coupling constants and the masses for the effective mean-field Lagrangian, and the effective interaction in the pairing channel. In most applications we have used the NL3 effective interaction [19] for the RMF Lagrangian. Properties calculated with NL3 indicate that this is probably the best effective interaction so far, both for nuclei at and away from the line of $\beta$-stability. For the pairing field we employ the pairing part of the Gogny interaction

$$
V^{p p}(1,2)=\sum_{i=1,2} e^{-\left(\left(\mathbf{r}_{1}-\mathbf{r}_{2}\right) / \mu_{i}\right)^{2}}\left(W_{i}+B_{i} P^{\sigma}-H_{i} P^{\tau}-M_{i} P^{\sigma} P^{\tau}\right)
$$

with the set D1S [20] for the parameters $\mu_{i}, W_{i}, B_{i}, H_{i}$ and $M_{i}(i=1,2)$. This force has been very carefully adjusted to the pairing properties of finite nuclei all over the periodic table. In particular, the basic advantage of the Gogny force is the finite range, which automatically guarantees a proper cut-off in momentum space. The RHB model with the 
NL3+D1S effective interaction has been applied in studies of the neutron halo phenomenon in light nuclei [15], properties of light nuclei near the neutron-drip line [21, ground state properties of $\mathrm{Ni}$ and $\mathrm{Sn}$ isotopes [22], the deformation and shape coexistence phenomena that result from the suppression of the spherical $\mathrm{N}=28$ shell gap in neutron-rich nuclei [23], the structure of proton-rich nuclei and the phenomenon of ground state proton emission 18,24, 25. In particular, it has been shown that neutron radii, calculated with the RHB NL3+D1S model, are in excellent agreement with experimental data 21.22].

\section{NEUTRON DENSITIES FOR ATOMIC PNC EXPERIMENTS}

In this section we apply the relativistic Hartree-Bogoliubov model, with the NL3+D1S effective interaction, in the calculation of ground-state proton and neutron densities of Cs, $\mathrm{Ba}, \mathrm{Yb}$ and $\mathrm{Pb}$ isotopes. These elements have been considered for measurements of isotope ratios of observables in atomic PNC experiments [1, 5, 8, 10].

Due to the large uncertainties in the experimental data on ground-state isovector properties, most of the effective interactions used in the non-relativistic Hartre-Fock model, and the effective Lagrangians of the relativistic mean-field model, have not been specifically designed to describe neutron density distributions. Only more recently have isovector properties been included in the set of data on which the effective interactions are adjusted. In the relativistic mean-field model, perhaps the most accurate set of meson masses and meson nucleon coupling constants is NL3 [19]. In Ref. [26] this effective force has been used in the relativistic mean-field + BCS model to calculate the ground-state properties of 1315 even-even nuclei with $10 \leq Z \leq 98$. The calculated quantities include the total binding energies, rms radii, quadrupole and hexadecupole deformations. In order to illustrate the quality of the results that are obtained with the NL3 interaction, in Fig. 11 we display the binding energies and isotope shifts of the charge radii of $\mathrm{Pb}$ nuclei. The calculation has been performed with the RHB model, and the theoretical values are compared with the experi-

mental data on binding energies [27] and charge radii [28]. The agreement between theory 
and experiment is very good, and we notice in particular that the calculated radii reproduce the anomalous kink in the isotopic shifts at ${ }^{208} \mathrm{~Pb}$ [28,29]. The NL3 effective force has also been used in studies of properties of excited states. By using the time-dependent relativistic mean-field model [30], and the relativistic random-phase approximation [31], it has been shown that a self-consistent calculation with the NL3 interaction reproduces the excitation energies of giant resonances in spherical nuclei. Particularly relevant for our present study are isovector giant resonances. In Fig. 2 we show the isovector dipole and monopole strength distributions in ${ }^{208} \mathrm{~Pb}$, calculated with the NL3 effective interaction in the relativistic RPA framework. The calculated peak energy for the dipole resonance $E_{p}=12.9 \mathrm{MeV}$ has to be compared with the experimental value of the excitation energy $13.3 \pm 0.1 \mathrm{MeV}$ [32]. The calculated isovector monopole strength is much more fragmented, but the centroid is also in good agreement with the experimental value for the IV GMR: $26 \pm 3 \mathrm{MeV}$ [33]. In Fig 3 we plot the binding energies, quadrupole deformations, and isotope shifts of the charge radii for the $\mathrm{Yb}, \mathrm{Ba}$ and $\mathrm{Cs}$ isotope chains. The results of the self-consistent RHB NL3+D1S calculations are compared with the available experimental data 27,28,34. The overall agreement between the theoretical predictions and experimental data is excellent. The results shown in Figs. 1 3 demonstrate that the RHB model with the NL3+D1S effective interaction is adequate for the calculation of ground-state neutron densities.

The self-consistent RHB neutron and proton ground-state density distributions for the isotopes ${ }^{202,208,214} \mathrm{~Pb}$ are shown in Fig. 4. We will eventually compare the calculated rms radii for ${ }^{202-214} \mathrm{~Pb}$, but here we notice the trend: the neutron radii increase with neutron number, while the neutron densities in the bulk region do not change much, except for some shell effects in the central region. The proton densities on the other hand, decrease in the bulk region as more neutrons are added on the surface. This effect, of course, has its origin in the symmetry energy. Therefore, one also observes an increase of proton radii, but much less pronounced than in the case of neutron densities. The difference in the trend of neutron and proton radii is emphasized in the inserts of Fig. 4, where the densities in the surface region are plotted on the logarithmic scale. 
In Ref. [10] it was suggested that the wide range of stable isotopes of $\mathrm{Yb}$ might be used for a comparison of PNC observables. These nuclei are generally deformed and therefore, in order to represent graphically the deformed densities, the density functions are decomposed in multipole moments

$$
\rho(\mathbf{r})=\frac{1}{\sqrt{4 \pi}} \sum_{L} \rho_{L}(r) Y_{L 0}(\theta, \phi) .
$$

The monopole $L=0$, quadrupole $L=2$, and hexadecupole $L=4$ components of the neutron density in ${ }^{174} \mathrm{Yb}$ are shown in Fig. 5. The deformations correspond to the neutron quadrupole moment $Q_{n}=11.02 b$, and the neutron hexadecupole moment $H_{n}=0.067 b^{2}$. Since the hexadecupole deformations are generally small, in the following figures we compare only the monopole and quadrupole components of the ground-state proton and neutron densities. These are shown in Fig. 6 for the even-N Yb isotopes. The deformations generally decrease with increasing the neutron number (see the $\beta_{2}$ values in Fig. 3). The difference between the quadrupole components of the proton and neutron density is small for all $\mathrm{Yb}$ nuclei in the sequence $N=98-106$. The proton and neutron densities differ much more in the spherical part of the distribution, not only on the surface (different radii), but especially in the bulk region. Similar features are observed for the Ba (Fig. 7) and Cs (Fig. 8) isotopes. These nuclei are less deformed, and the spherical approximation for the densities will work better than in the case of the $\mathrm{Yb}$ isotopes. Correspondingly, the differences in the quadrupole components of the neutron and proton densities are smaller. The monopole components of these densities, however, display very different behavior both in the bulk and the surface region.

The isotope shifts for the neutron radii of the ground-state density distributions of Cs, $\mathrm{Ba}, \mathrm{Yb}$ and $\mathrm{Pb}$ nuclei are shown in Fig. 9. In all four cases a uniform increase of the neutron radii is observed. The differences between neutron and proton rms radii are plotted in Fig. 10, as function of the number of neutrons. The differences are of the order of $0.2-0.3$ fm and, of course, increase with the neutron number. The increase, however, is not very steep. For the Yb nuclei, for example, $r_{n}-r_{p}$ increases by less than $0.05 \mathrm{fm}$ between $N=100$ 
and $N=106$. The ratios $r_{n} / r_{p}$ are compared in Fig. 11. We notice that while the isotope trend is similar, the actual values of $r_{n} / r_{p}$ calculated with the RHB NL3+D1S model are considerably larger than those obtained in the Hartre-Fock Skyrme calculations for Pb [8] and Cs [2.9]. In particular, for ${ }^{133} \mathrm{Cs}$ we calculate $r_{n} / r_{p}=1.046$, as compared to the HartreFock values: 1.022 for SkM*, and 1.016 for SkIII. An approximate estimate for the nuclear structure correction (4) to the weak charge has been derived in Ref. [2]

$$
\Delta Q_{W}^{n-p} \approx N(Z \alpha)^{2}(.221 \epsilon) / q_{p}
$$

where the small parameter $\epsilon$ is defined by

$$
\left(r_{n}^{2} / r_{p}^{2}\right) \equiv 1+\epsilon
$$

The RHB NL3 value for $r_{n} / r_{p}$ implies, therefore, that the nuclear structure correction to the weak charge can be more than a factor 2 larger than the estimate given in [2], bringing the value of $\Delta Q_{W}^{n-p}$ close to the uncertainties of atomic theory calculations. On the other hand, the results shown in Figs. 10 and 11 indicate that the changes in the neutron distribution parameters along the isotope chains should not present a limitation on the accuracy with which the Standard model can be tested in measurements of isotope ratios of PNC observables.

\section{ACKNOWLEDGMENTS}

This work has been supported in part by the Bundesministerium für Bildung und Forschung under project 06 TM 979, by the Deutsche Forschungsgemeinschaft, and by the Gesellschaft für Schwerionenforschung (GSI) Darmstadt. We thank Andreas Wandelt for the relativistic RPA calculation of isovector monopole and dipole strength distributions in ${ }^{208} \mathrm{~Pb}$.

[1] M. J. Ramsey-Musolf, Phys. Rev. C 60, 015501 (1999). 
[2] S. J. Pollock and M. C. Welliver, Phys. Lett. 464B, 177 (1999).

[3] S.C. Bennett and C.E. Wieman, Phys. Rev. Lett. 82, 2484 (1999).

[4] V.A. Dzuba, V.V. Flambaum, and I.B. Khriplovich, Z. Phys. D 1, 243 (1986); C. Monroe, W. Swann, H. Robinson, and C.E. Wieman, Phys. Rev. Lett. 65, 1571 (1990).

[5] E.N. Fortson, Y. Pang, and L. Wilets Phys. Rev. Lett. 65, 2857 (1990).

[6] T. Suzuki et al., Phys. Rev. Lett. 75, 3241 (1995).

[7] A. Krasznahorkay et al., Phys. Rev. Lett. 82, 3216 (1999).

[8] S.J. Pollock, E.N. Fortson, and L. Wilets, Phys. Rev. C 46, 2587 (1992).

[9] P.Q. Chen and P. Vogel, Phys. Rev. C 481392 (1993).

[10] D. DeMille, Phys. Rev. Lett. 74, 4165 (1995).

[11] C. J. Horowitz, S. J. Pollock, P. A. Souder, R. Michaels, Los Alamos preprint nucl-th/9912038.

[12] B.D. Serot and J.D. Walecka, Adv. Nucl. Phys. 16, 1 (1986); Int. J. Mod. Phys. E6, 515 (1997).

[13] P. Ring, Progr. Part. Nucl. Phys. 37, 193 (1996).

[14] J. Meng and P. Ring, Phys. Rev. Lett. 77, 3963 (1996).

[15] W. Pöschl, D. Vretenar, G.A. Lalazissis, and P. Ring, Phys. Rev. Lett. 79, 3841 (1997).

[16] D. Vretenar, P. Finelli, A. Ventura, G.A. Lalazissis, and P. Ring, Los Alamos preprint nuclth/9911024, Phys. Rev. C in print.

[17] Y.K. Gambhir, P. Ring and A. Thimet, Ann. Phys. (N.Y.) 198, 132 (1990).

[18] G.A. Lalazissis, D. Vretenar, and P. Ring, Nucl. Phys. A650, 133 (1999).

[19] G. A. Lalazissis, J. König and P. Ring; Phys. Rev. C55, 540 (1997). 
[20] J. F. Berger, M. Girod and D. Gogny; Nucl. Phys. A428, 32 (1984).

[21] G.A. Lalazissis, D. Vretenar, W. Pöschl, and P. Ring, Nucl. Phys. A632, 363 (1998).

[22] G.A. Lalazissis, D. Vretenar, and P. Ring, Phys. Rev. C 57, 2294 (1998).

[23] G.A. Lalazissis, D. Vretenar, P. Ring, M. Stoitsov, and L. Robledo, Phys. Rev. C 60, 014310 (1999).

[24] D. Vretenar, G.A. Lalazissis, and P. Ring, Phys. Rev. Lett. 82, 4595 (1999).

[25] G.A. Lalazissis, D. Vretenar, and P. Ring, Phys. Rev. C 60, 051302 (1999).

[26] G.A. Lalazissis, S. Raman and P. Ring, At. Data Nucl. Data Tables 71, 1 (1999).

[27] G. Audi and A. H. Wapstra, Nucl. Phys. A595, 409 (1995).

[28] E.W. Otten, in Treatise on Heavy-Ion Science, edited by D.A. Bromley (Plenum, New York, 1989) Vol 8, p. 515.

[29] M.M. Sharma, G.A. Lalazissis, and P. Ring, Phys. Lett. B317, 9 (1993).

[30] D. Vretenar, G.A. Lalazissis, R. Behnsch, W. Pöschl and P. Ring, Nucl. Phys. A 621 (1997) 853.

[31] D. Vretenar, A. Wandelt, and P. Ring, Los Alamos preprint nucl-th/0003041.

[32] J. Ritman et al., Phys. Rev. Lett. 70533 (1993).

[33] P. Gleissl, M. Brack, J. Meyer, and P. Quentin; Ann. Phys. (N.Y.) 197205 (1990).

[34] S. Raman, C.H. Malarkey, W.T. Milner, C.W. Nestor and P.H. Stelson, At. Data Nucl. Data Tables 36, 1 (1987). 


\section{Figure Captions}

FIG. 1. Binding energies (left), and isotope shifts of the charge radii (right) of $\mathrm{Pb}$ nuclei, calculated with the NL3 + Gogny D1S effective interaction. The theoretical values are compared with the experimental binding energies [27] and charge radii [28].

FIG. 2. The isovector dipole and monopole strength distributions in ${ }^{208} \mathrm{~Pb}$, calculated with the NL3 effective interaction in the relativistic RPA framework.

FIG. 3. Binding energies (left), quadrupole deformations (center), and isotope shifts of the charge radii (right), for the $\mathrm{Yb}, \mathrm{Ba}$ and $\mathrm{Cs}$ isotope chains. The results of the self-consistent $\mathrm{RHB}$ calculations are compared with the available experimental data [27, 34, 28].

FIG. 4. Self-consistent RHB neutron and proton ground-state density distributions for ${ }^{202,208,214} \mathrm{~Pb}$. In the inserts the same densities in the surface region are plotted on the logarithmic scale.

FIG. 5. The monopole, quadrupole and hexadecupole components of the self-consistent RHB neutron ground-state density distribution in the nucleus ${ }^{174} \mathrm{Yb}$.

FIG. 6. Monopole and quadrupole components of the self-consistent RHB neutron (solid line) and proton (dot-dashed line) ground-state density distributions for the even-N Yb isotopes.

FIG. 7. Same as in Fig. 6, but for the Ba isotopes.

FIG. 8. Same as in Fig. 6, but for the Cs nuclei.

FIG. 9. Isotope shifts for the neutron radii of the ground-state density distributions of Cs, Ba, $\mathrm{Yb}$ and $\mathrm{Pb}$ nuclei.

FIG. 10. Differences between neutron and proton radii of the ground-state density distributions for the $\mathrm{Cs}, \mathrm{Ba}, \mathrm{Yb}$ and $\mathrm{Pb}$ isotope chains. 
FIG. 11. Ratios of neutron to proton radii for the ground-state density distributions of the Cs, $\mathrm{Ba}, \mathrm{Yb}$ and $\mathrm{Pb}$ isotope chains. 

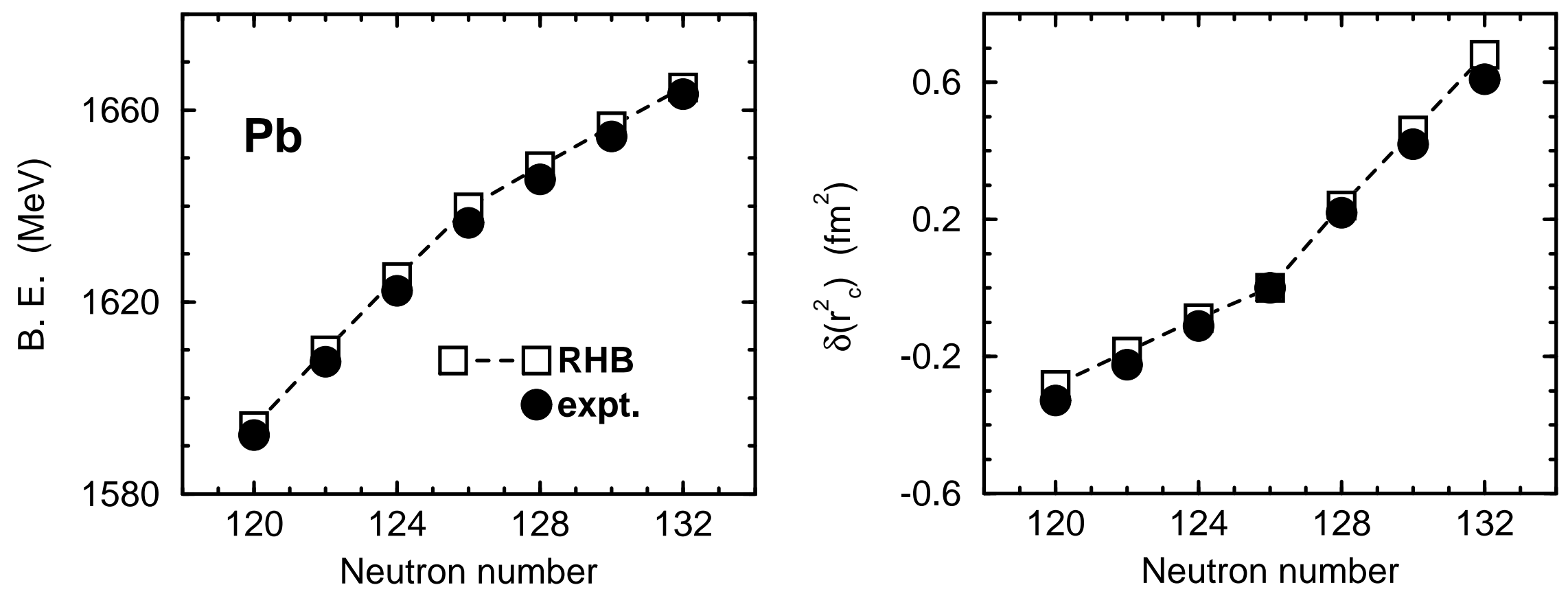

Fig. 1 

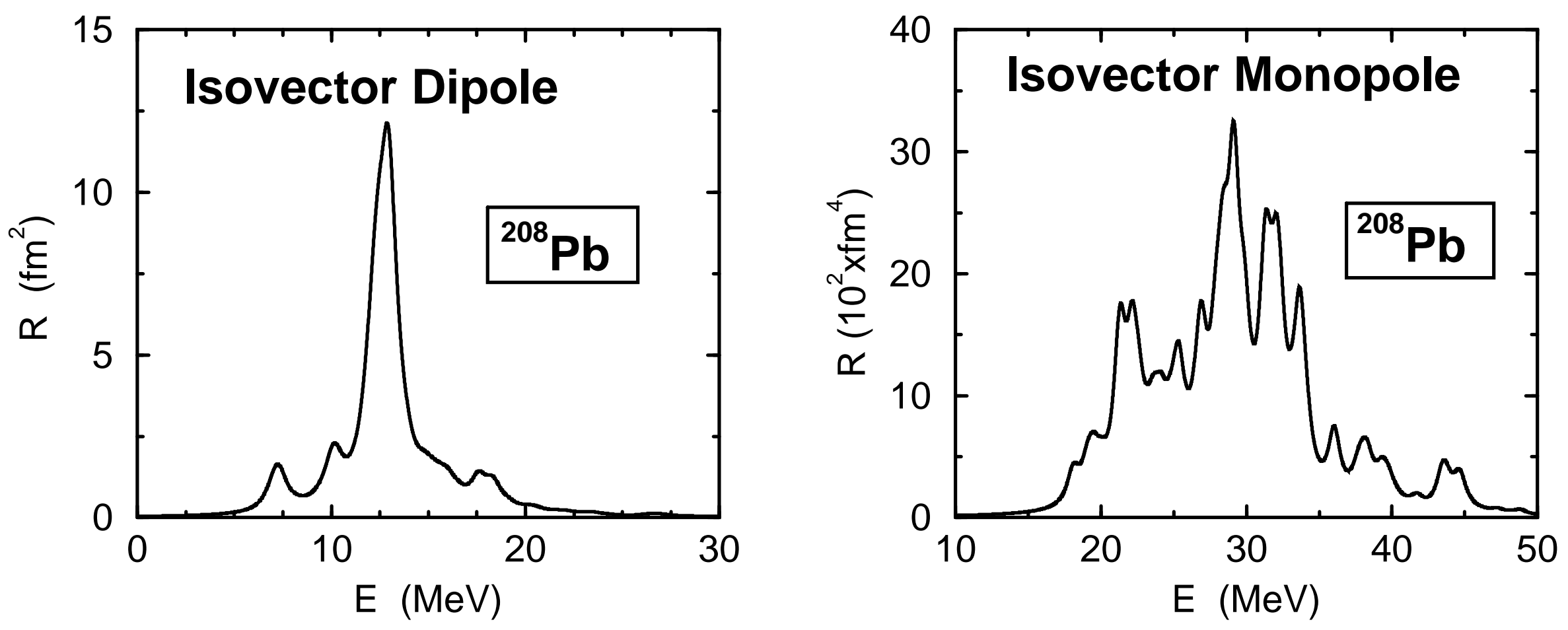

Fig. 2 

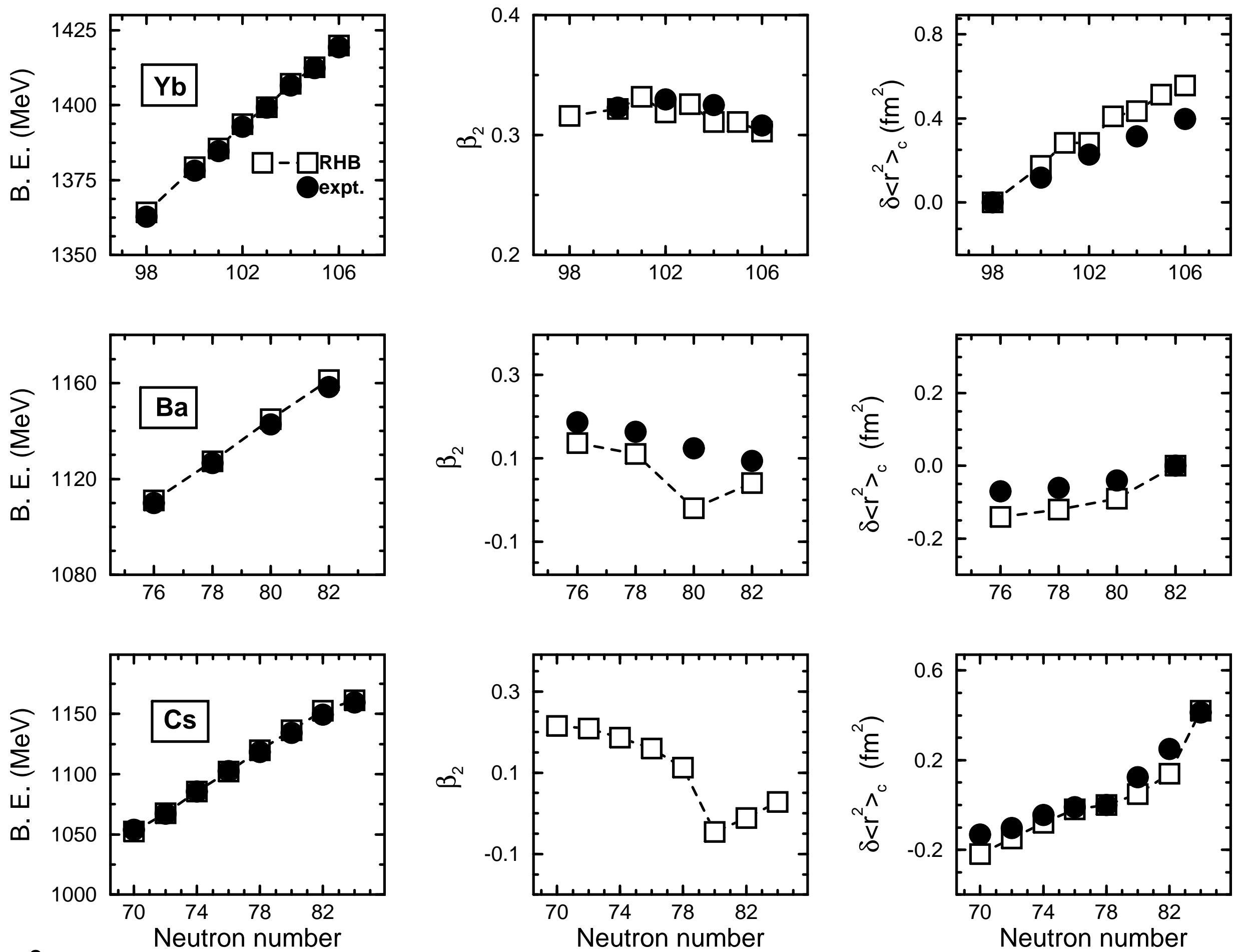

Fig. 3
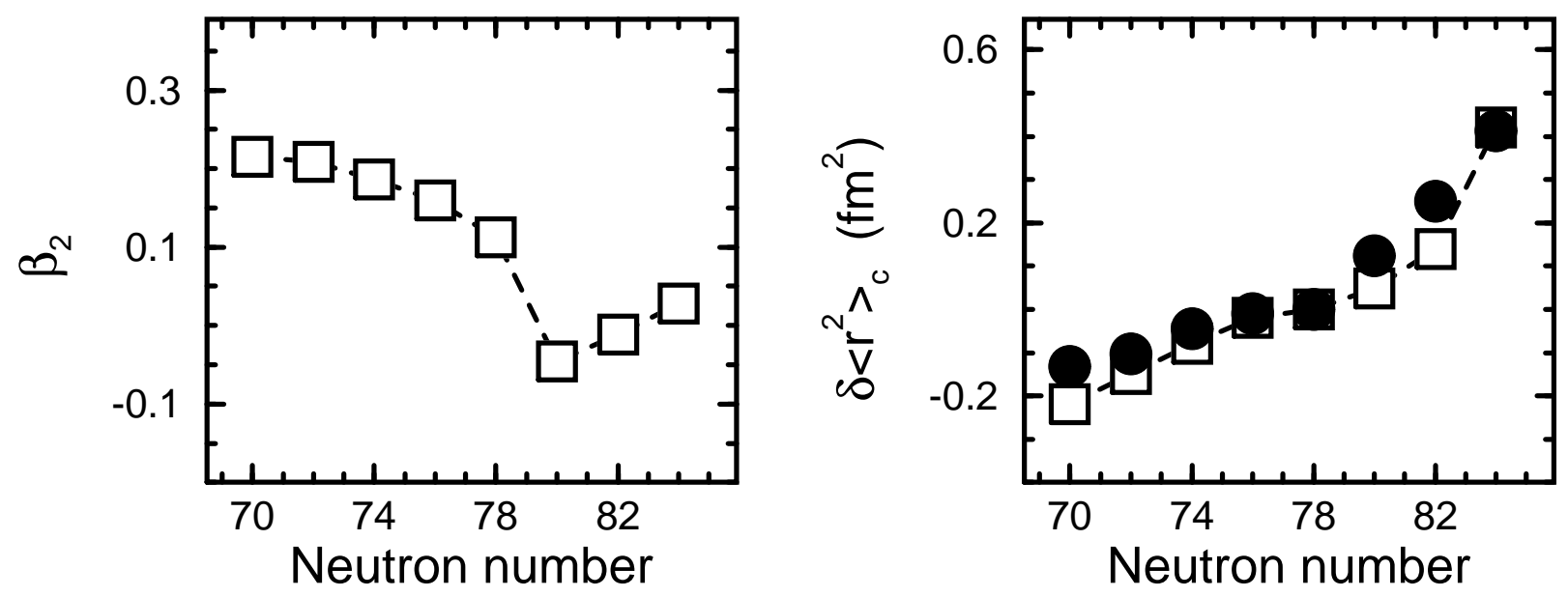

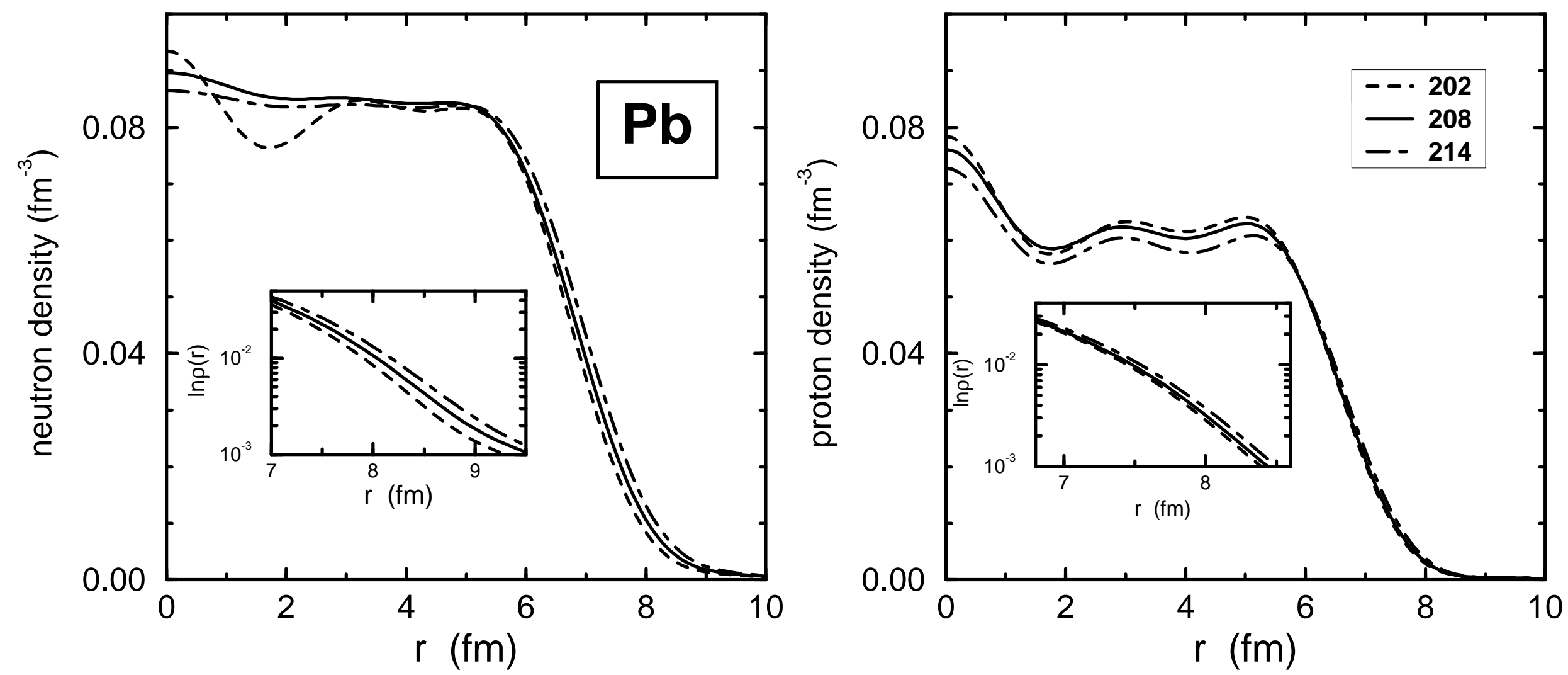

Fig. 4 


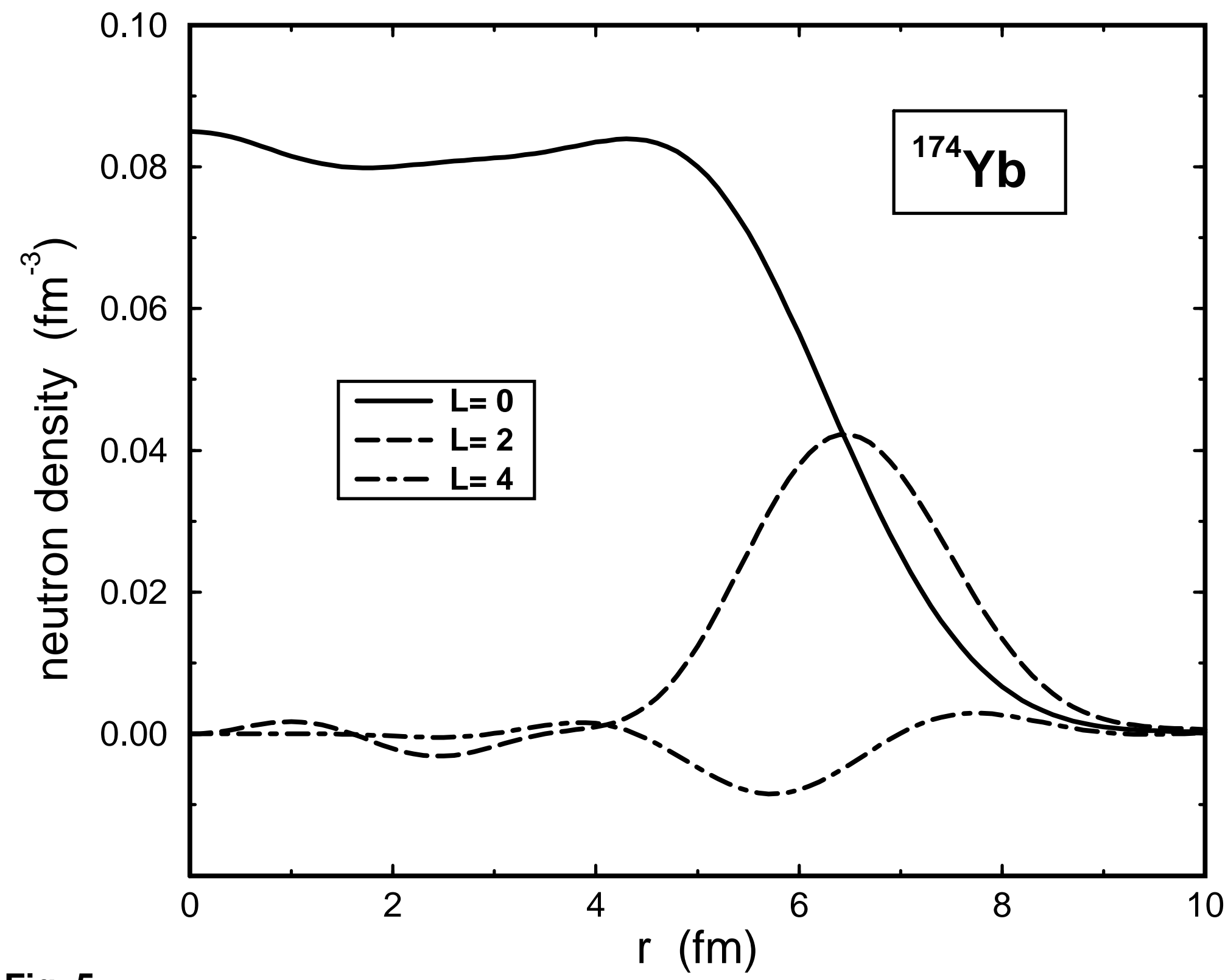

Fig. 5 

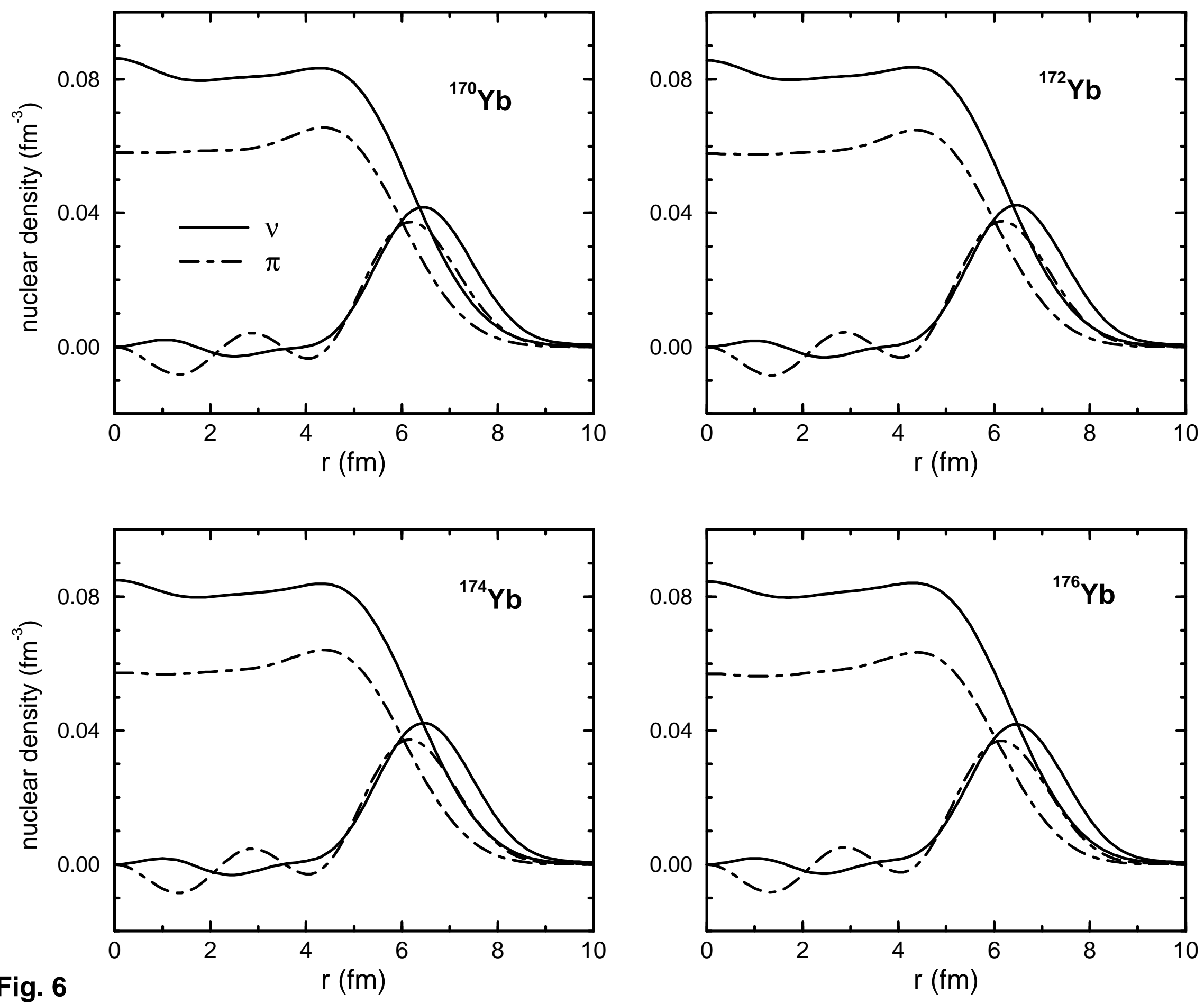

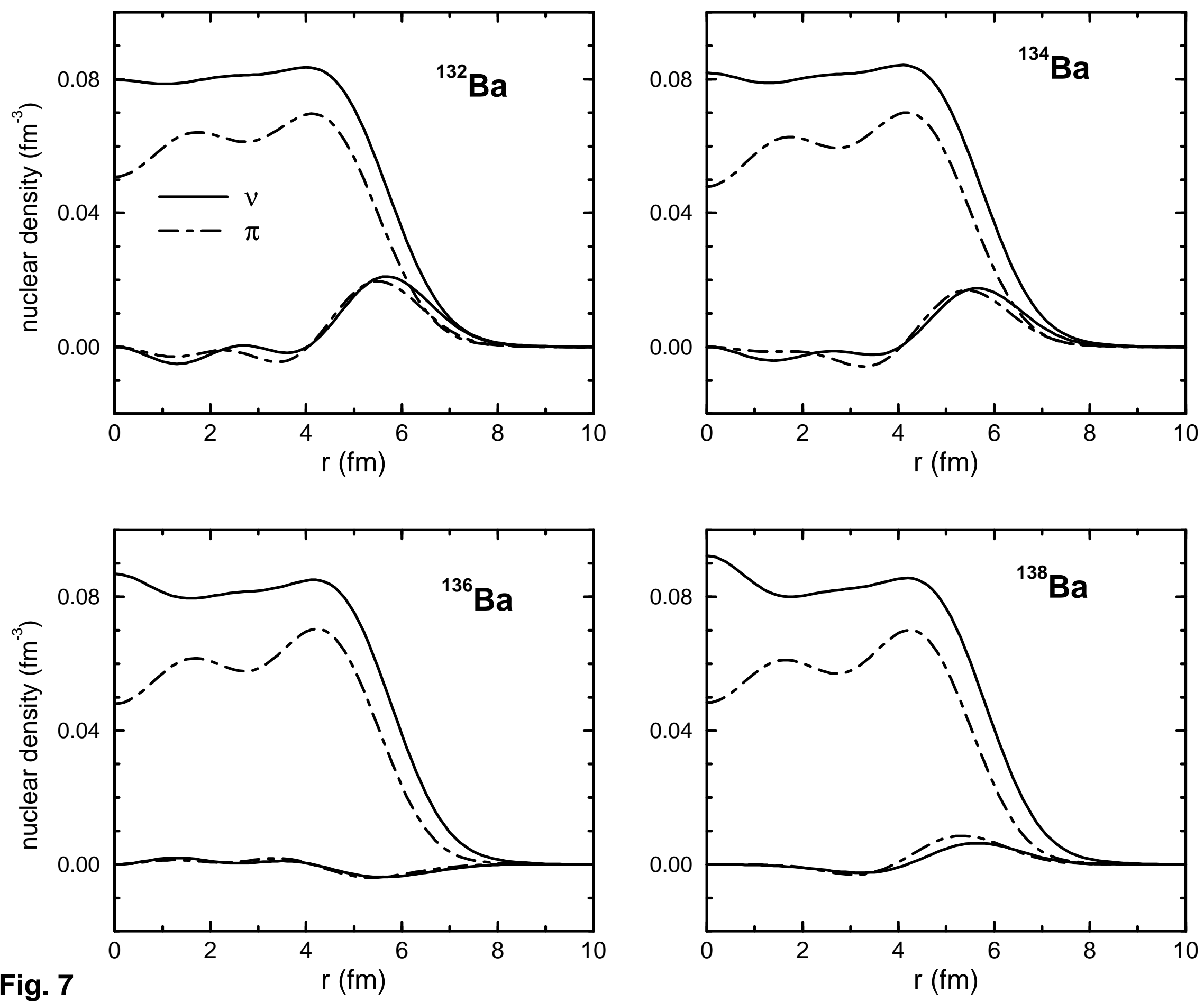

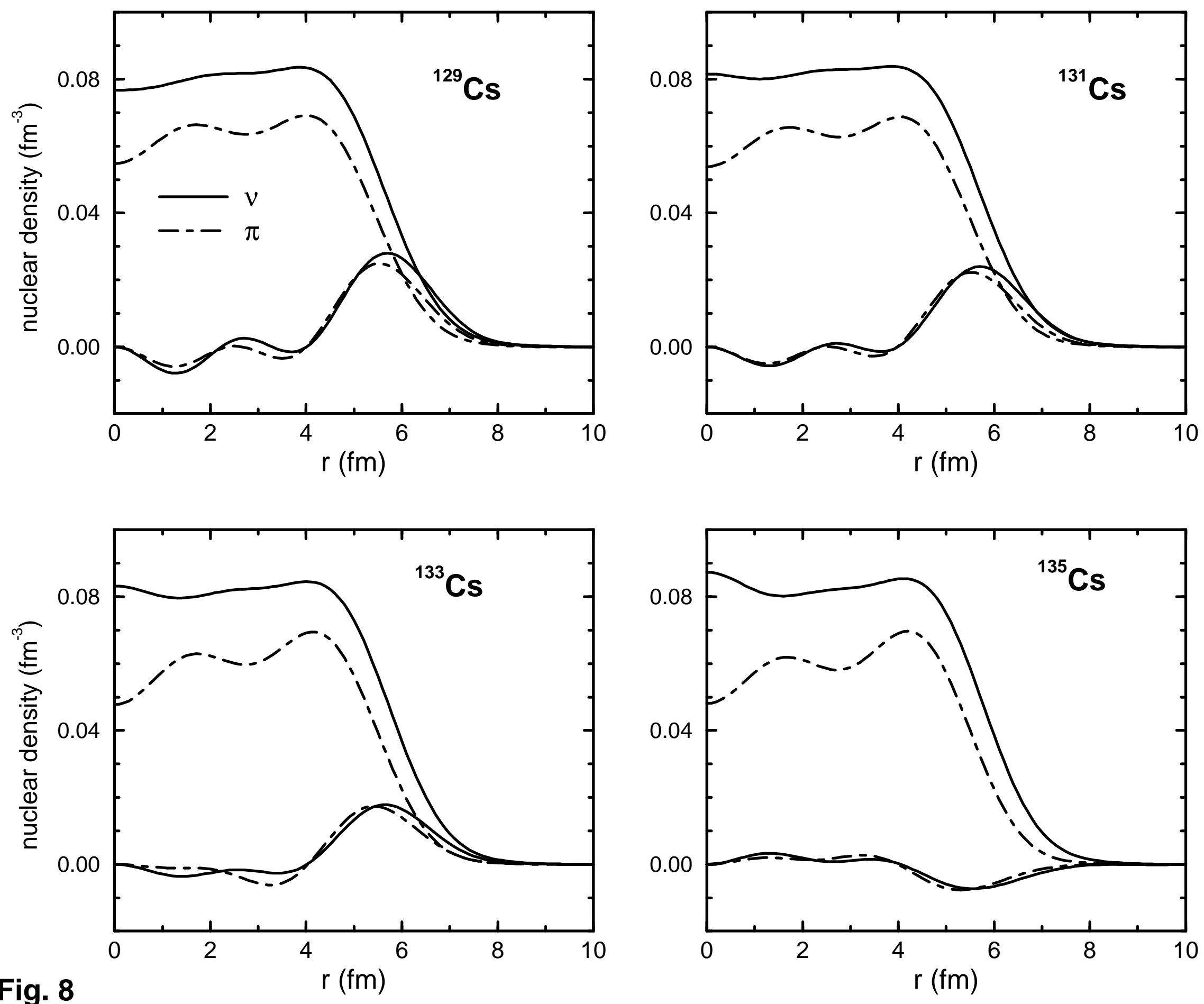

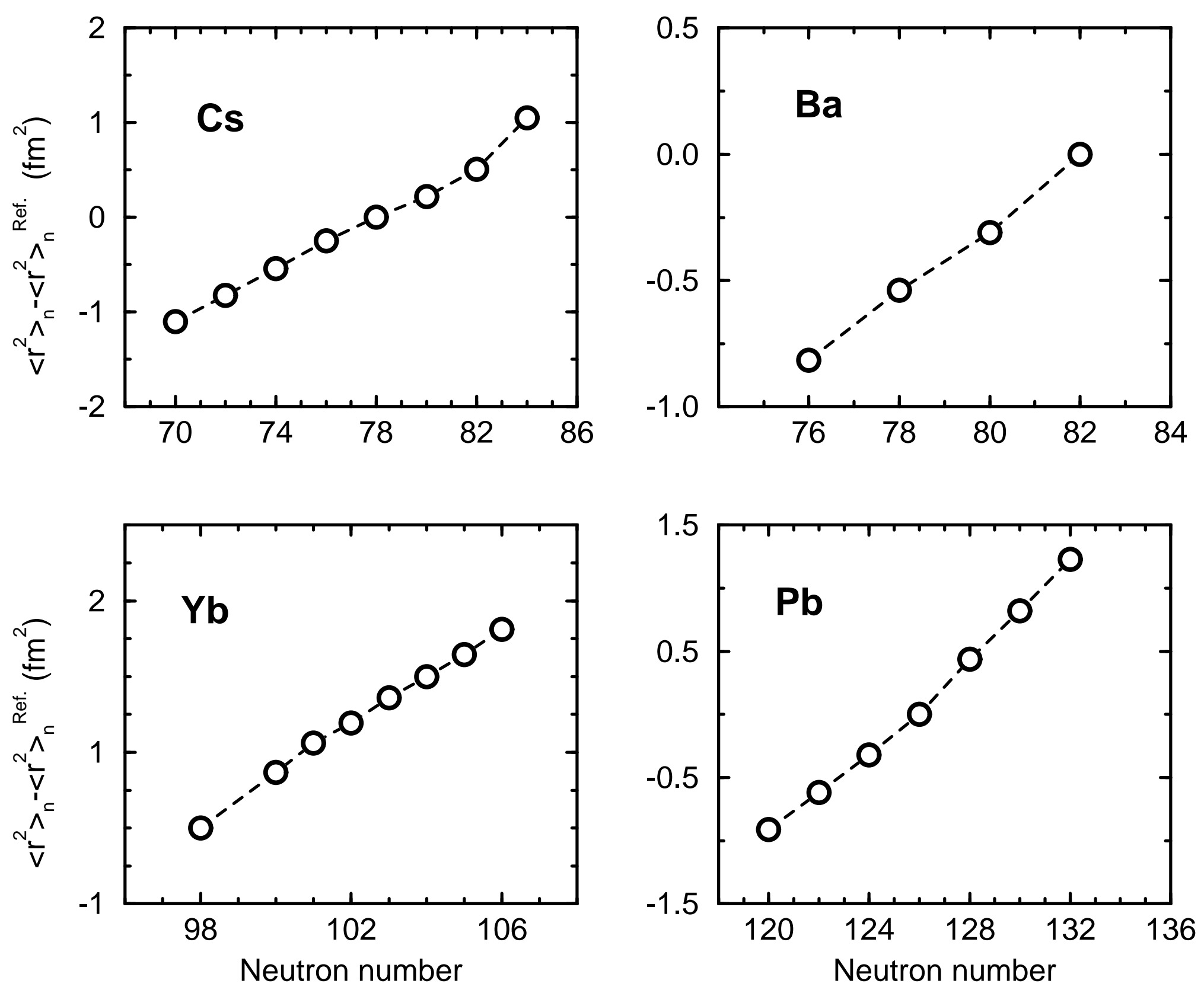

Fig. 9 

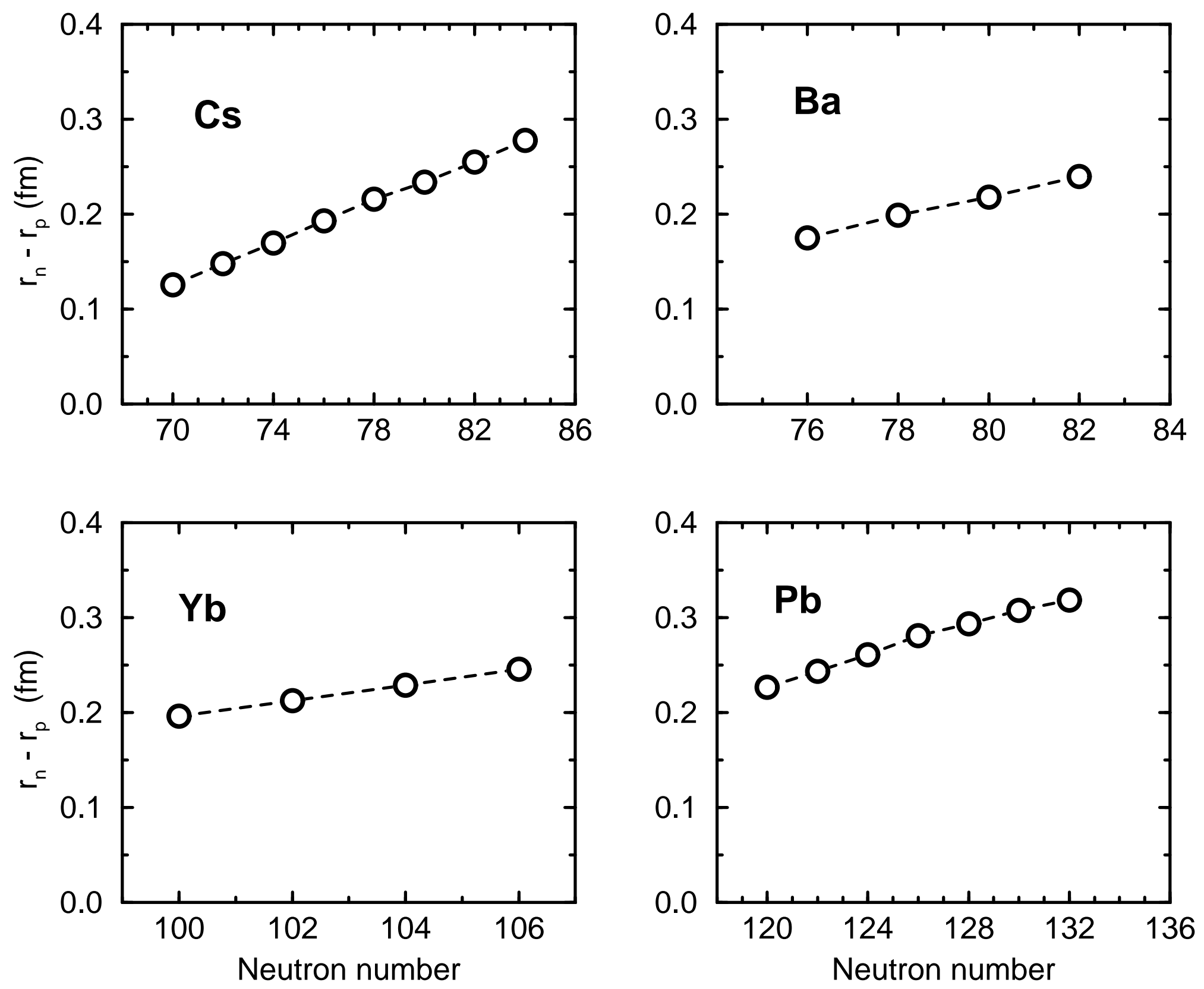

Fig. 10 

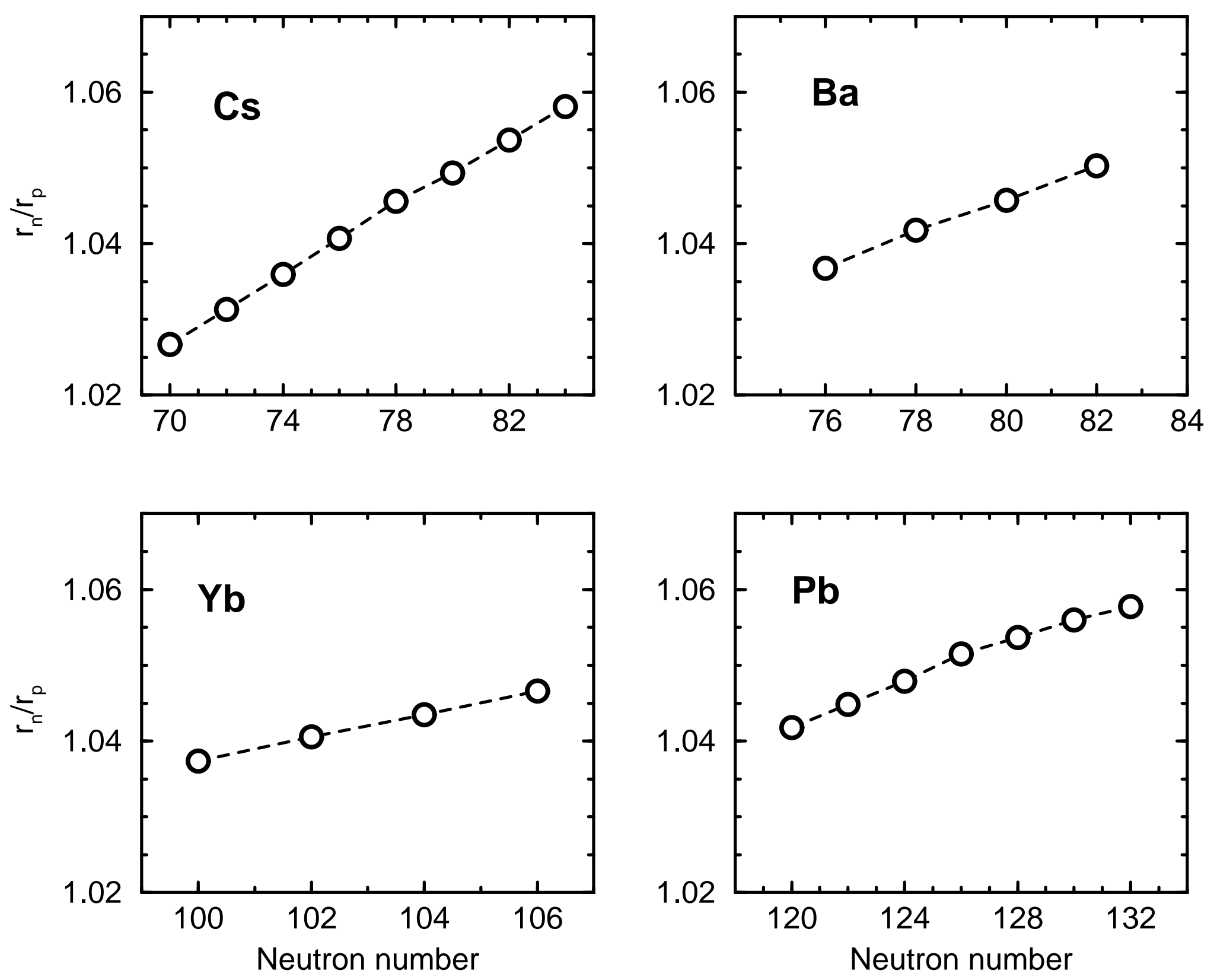

Fig. 11 